\title{
Characterization of Line-of-Sight and Non-Line-of-Sight Pseudorange Multipath Errors in Urban Environment for GPS and Galileo
}

\author{
Eustachio Roberto Matera (ABBIA), Axel Garcia-Pena (ENAC), Olivier Julien (ENAC), \\ Carl Milner (ENAC), Bertrand Ekambi (ABBIA)
}

Ecole Nationale de l'Aviation Civile (ENAC), ABBIA GNSS Technologies, Toulouse, France BIOGRAPHIES

Eustachio Roberto Matera is currently a PhD student at ENAC. He has obtained telecommunication engineer diploma in 2014 and a Master of Science in wireless communication in 2016 at Politecnico di Torino, Italy. He is currently studying signal processing algorithms and integrity monitoring techniques adapted to the navigation in urban areas. His thesis is funded by ABBIA GNSS Technologies.

Axel Garcia Pena is a researcher/lecturer with the SIGnal processing and NAVigation (SIGNAV) research group of the TELECOM lab of ENAC (French Civil Aviation University), Toulouse, France. His research interests are GNSS navigation message demodulation, optimization and design, GNSS receiver design and GNSS satellite payload. He received his double engineer degree in 2006 in digital communications from SUPAERO and UPC, and his PhD in 2010 from the Department of Mathematics, Computer Science and Telecommunications of the INPT (Polytechnic National Institute of Toulouse), France.

Olivier Julien is the head of the SIGnal processing and NAVigation (SIGNAV) research group of the TELECOM lab of ENAC (French Civil Aviation University), Toulouse, France. His research interests are GNSS receiver design, GNSS multipath and interference mitigation, and interoperability. He received his engineer degree in 2001 in digital communications from ENAC and his PhD in 2005 from the Department of Geomatics Engineering of the University of Calgary, Canada.

Carl Milner is an Assistant Professor within the Telecom Lab at the Ecole Nationale de l'Aviation Civile. He has a Masters degree in Mathematics from the University of Warwick, a $\mathrm{PhD}$ in Geomatics from Imperial College London and has completed the graduate trainee programme at the European Space Agency. His research interests include GNSS augmentation systems, integrity monitoring, air navigation and applied mathematics.

Bertrand Ekambi graduated by a Master in Mathematical Engineering in 1999. Since 2000, he is involved in the main European GNSS projects: EGNOS and GALILEO. He is the founder manager of ABBIA GNSS Technologies, a French SME working on Space Industry, based in Toulouse, France.

\footnotetext{
ABSTRACT

An increasing number of new applications require an accurate positioning even in urban environments; however, in such environments, especially in urban canyons, GNSS positioning is challenged to meet the applications' demanded accuracy and reliability. In fact, in order to obtain an optimal and reliable position estimate using GNSS, it is necessary to have an accurate model of the pseudorange and pseudorange rate error terms' distributions.

This work focuses thus its attention on the statistical characterization of the pseudorange measurements' multipath error component by proposing a methodology to obtain such characterization: isolation of the multipath error component from the use of a reference station, to eliminate ionospheric error terms, and from a filtering process, to eliminate the receiver clock bias. The proposed methodology has been adapted to include dual constellation measurements in the L1 band, GPS L1 C/A and Galileo E1 OS signal measurements, as an evolution to the previous method presented by the authors in [1].
} 
Moreover, in order to obtain a reliable classification of the signal reception conditions, Lion-of-Sight (LOS) and Non LOS (NLOS), which will allow a finer characterization of the multipath error component, this work introduces the use of an upward looking camera with a wide Field-of-View (FOV), a fisheye camera: the satellites are projected on the pictures taken by the camera allowing to observe which satellites are obstructed by the scenario obstacles (buildings, trees, etc).

The proposed methodology is applied to real measurements obtained from a data campaign conducted in Toulouse urban area with a U-Blox receiver with its antenna and a fisheye camera mounted on the roof of a car. The pseudorange measurements are classified by the signal $C / N_{0}$ and by the elevation angle between the satellites and the receiver, which are common signal characteristics influencing the multipath error component impact on the pseudorange measurement. Additionally, the performance assessment of each parameter in terms of signal reception conditions classification between LOS and NLOS has determined the upper hand of the $C / N_{0}$ parameter.

\section{INTRODUCTION}

Nowadays, navigation systems integrating at least an inertial measurement unit (IMU) and GNSS signal processing units are becoming the fundamental baseline platform for mass-market user devices. Such platforms aim at combining reasonably low-cost hardware with the provision of the highest possible positioning accuracy, availability and reliability.

Despite being equipped with an IMU, these hybrid systems will still rely on GNSS measurements for correcting IMU increasing-in-time bias errors. Therefore, in order to obtain an optimal and reliable position estimate, a system will have to have access to an accurate assessment of the GNSS measurements even in difficult environments such as urban/indoor scenarios. More specifically, urban environments present a significant challenge for modern Global Navigation Satellite Systems (GNSS) since the satellite signals reception conditions are very harsh: surrounding obstacles reflect/diffract the satellites transmitted signals, creating the multipath phenomenon, or even block the LOS signal, creating non-line-of-sight (NLOS) received satellite signal conditions. As a result, the pseudorange measurements will be significantly erroneous and different from their expected model in open sky conditions, and thus the final position estimation will have larger errors than in open sky conditions. Nevertheless, a good characterization of these effects could help mitigate the loss of positioning performance (such as accuracy or reliability/integrity) with respect to the error in open-sky environments. In conclusion, it is necessary provide an accurate pseudo-range error distribution mathematical model to the system to obtain the desired positioning performances in any type of environment.

The multipath effect on GNSS receivers has been theoretically studied in the literature with its main focus on fixed position test conditions. These studies have analyzed the effects of multipath on the pseudorange measurements [2][3]. Moreover, multiple studies based on simulations have also been conducted to address the multipath impact on the code phase and the carrier phase measurements [4][5]; and even real pseudorange measurement analysis and characterization has already been proposed by the authors in [1]: a pseudorange multipath error isolation model and a multipath error characterization method, based on signals $C / N_{0}$ and satellite signal elevation angle.

In this paper, the general goal is thus to improve the methodology provided in [1] and to apply the complete methodology in a real data from a measurement campaign. The main improvements provided by this work with respect to [1] are:

a) To exploit a dual constellation GPS/Galileo pseudorange measurements in the L1 frequency band instead of single GPS L1 C/A measurements,

b) To introduce a NLOS/LOS characterization of the multipath error component based on a NLOS/LOS classification algorithm. The classification algorithm consists in an upward-looking fish-eye camera and specific image-processing software allowing to separate the satellite signals received in LOS and NLOS conditions.

Therefore, the methodology proposed in this work to characterize the multipath error component impact on the pseudorange measurement consists of the following 3 steps:

1- A multipath error component isolation method from the L1 band pseudorange measurement is applied; this method is based on [1] with some modifications to include dual constellation measurements.

2- A NLOS/LOS signal conditions classification is performed using an upward-looking fish-eye camera and an image processing method. 
3- A multipath error characterization process is conducted: the probability density function is estimated and classified as a function of the LOS/NLOS signal conditions, the satellite $C / N_{0}$ and the elevation angle between the satellite and the receiver.

Finally, a real application example of the proposed methodology is conducted based on a data measurement campaign performed in Toulouse urban area; the LOS and NLOS classification performance of each parameter has been assessed

The paper is organized as follows. Section 2 describes the multipath error isolation method. Section 3 describes the multipath error component isolation for dual constellation. Section 4 describes the multipath error component characterization process and the NLOS/LOS classification algorithm. Section 5 describes the experimental setup used to conduct the signal test campaign as well as the vehicle trajectory inside Toulouse urban area. The results obtained are presented and analyzed in Section 6. Finally, a discussion on the methodology used is made and conclusions are given about the article's main results regarding their exploitation for GNSS vehicle applications.

\section{MULTIPATH ERROR COMPONENT ISOLATION METHOD}

This section presents the theoretical fundamentals on which the multipath error isolation method is based. More details on multipath error isolation method are provided in [1].

\subsection{Fundamental idea}

The code pseudorange measurement obtained by a user receiver, $x$, from satellite $i$ at a given instant $t$ can be accurately modelled as [1]:

where

$$
\begin{gathered}
P_{x}^{i}(t)=R_{x}^{i}(t)+b_{x}(t)-b_{s v}^{i}(t)+I_{x}^{i}(t)+T_{x}^{i}(t)+M_{x}^{i}(t)+h_{x}(t)+ \\
+h_{s v}^{i}(t)+\eta_{x}^{i}(t)
\end{gathered}
$$

- $\quad R_{x}^{i}(t)$ is satellite-to-receiver range at time $t$

$\sqrt{\left(x_{s v}^{i}(t)-x(t)\right)^{2}+\left(y_{s v}^{i}(t)-y(t)\right)^{2}+\left(z_{s v}^{i}(t)-z(t)\right)^{2}}$ it is the effective range between the satellite and the receiver at epoch $t$, which could be used for positioning purpose.

- $\quad(x, y, z)$ are the receiver true coordinates

- $\quad\left(x_{s v}^{i}, y_{s v}^{i}, z_{s v}^{i}\right)$ are the satellite $i$ true coordinates

- $\quad c$ is the speed of light

- $\quad b_{x}$ is the receiver clock bias

- $\quad b_{s v}^{i}$ is the satellite $i$ clock bias

- $\quad I_{x}^{i}$ is the ionospheric delay from satellite $i$ measurement

- $\quad T_{x}^{i}$ is the atmospheric delay from satellite $i$ measurement

- $\quad M_{x}^{i}$ is the code multipath error component of the user from satellite $i$ measurement

- $\quad h_{x}$ is the receiver hardware bias

- $\quad h_{x}^{i}$ is the satellite $i$ hardware bias

- $\quad \eta_{x}^{i}$ is the random measurement noise of the user from satellite $i$ measurement

The proposed method consists thus in isolating, as much as possible, the multipath error component from the other pseudorange measurement terms. The procedures employed for the removal of the different terms depend on the nature of each term:

- The satellite-to-receiver range can be easily subtracted if the receiver and satellite position are known.

- The removal of all atmospheric and satellite-dependent elements can be obtained by differencing the pseudorange measurements with the measurements of a nearby reference station.

- The receiver clock and hardware biases can be estimated first and remover later.

\subsection{Detailed Method}

A method with three steps was described in [1] to isolate the multipath error component from the other terms of the pseudorange measurement. These three steps are summarized in the next three sections.

\subsection{1 "Range-Free" Measurements}

The first step of the multipath error isolation is to obtain a pseudorange residual which contains only the pseudorange error terms also called "range-free" pseudorange residual or just residual (per satellite). This 
residual can be easily determined by differencing the true receiver-to-satellite range (also called effective range), and the pseudorange measurement, equation 2.1. The mathematical expression is given in 2.2:

$$
\Delta_{u}^{\mathrm{i}}(t)=b_{u}(t)-b_{s v}^{i}(t)+E_{u}^{i}(t)+I_{u}^{i}(t)+T_{u}^{i}(t)+M_{u}^{i}(t)+h_{u}(t)+h_{s v}^{i}(t)+\eta_{u}^{i}(t)
$$

where $E_{u}^{i}$ is the error projected on the pseudorange domain due to the satellite $i$ position estimation error. The same can be done to obtain "range-free" reference station pseudorange residuals (see equation 2-3):

$$
\Delta_{s t a}^{\mathrm{i}}(t)=b_{s t a}(t)-b_{s v}^{i}(t)+E_{s t a}^{i}(t)+I_{s t a}^{i}(t)+T_{s t a}^{i}(t)+M_{s t a}^{i}(t)+h_{s t a}(t)+h_{s v}^{i}(t)+\eta_{s t a}^{i}(t)
$$

where

- $\quad b_{\text {sta }}$ is the reference station clock bias

- $\quad E_{\text {sta }}^{i}$ is the reference station pseudorange error projected on the pseudorange domain due to the satellite $i$ position estimation error

- $\quad M_{\text {sta }}^{i}$ is the code multipath error component of the reference station from satellite $i$ measurement

- $\quad h_{\text {sta }}$ is the reference station receiver hardware bias

- $\quad \eta_{\text {sta }}^{i}$ is the random measurement noise of the reference station from satellite $i$ measurement

\subsubsection{Pseudorange residual difference}

The second step of the proposed method consists in removing the impairments of the user receiver pseudorange residual which are common to the reference station ones. The removal is achieved by differencing the user "range free" measurement residual $\Delta_{u}^{\mathrm{i}}(t)$ from the "range free" reference station pseudorange residual $\Delta_{s t a}^{\mathrm{i}}(t)$ as presented in equation $2-4$.

$$
\begin{gathered}
\epsilon^{i}(t)=\Delta_{u}^{\mathrm{i}}(t)-\Delta_{\text {sta }}^{\mathrm{i}}(t)= \\
=b_{u}(t)-b_{\text {sta }}(t)+\delta_{S T C E}^{i}(t)+M_{u}^{i}(t)-M_{\text {sta }}^{i}(t)+h_{u}(t)-h_{s t a}(t)+\eta_{u}^{i}(t)-\eta_{s t a}^{i}(t) \\
\delta_{S T C E}^{i}(t)=E_{u}^{i}(t)-E_{\text {sta }}^{i}(t)+I_{u}^{i}(t)-I_{\text {sta }}^{i}(t)+T_{u}^{i}(t)-T_{\text {sta }}^{i}(t)
\end{gathered}
$$

Where:

- $\quad \delta_{S T C E}^{i}(t)$ is space-time correlated residual errors of satellite $i$ between the user receiver and the reference station

The resulting term, $\epsilon^{i}(t)$, is denoted as the pseudorange residual difference and is dominated by five factors:
a. the vehicle-reference station receiver clock difference, $\left(b_{u}(t)-b_{\text {sta }}(t)\right)$
b. the space-time correlated residual errors, $\delta_{S T C E}^{i}(t)$;
c. the user receiver multipath error component, $M_{u}^{i}(t)$ and the reference station multipath error component, $M_{\text {sta }}^{i}(t)$;
d. the receivers' bias hardware term, $h_{u}(t)-h_{\text {sta }}(t)$;
e. the user receiver noise, $\eta_{u}^{i}(t)$ and the reference station receiver noise, $\eta_{s t a}^{i}(t)$.

The differential measurement can be simplified into (2-6) as justified in [1]:

$$
\epsilon^{i}(t) \approx b h_{u}(t)+M_{u}^{i}(t)+\eta_{u}^{i}(t)-\eta_{s t a}^{i}(t) \approx b h_{u}(t)+M N_{u}^{i}(t)
$$

where

- $\quad$ the reference station multipath error component, $M_{\text {sta }}^{i}(t)$, as well as the space-time correlated residual errors, $\delta_{S T C E}^{i}(t)$, are assumed to be negligible with respect to $M_{u}^{i}(t)$.

- the reference station thermal noise is assumed to be negligible with the respect to the receiver under test noise. Moreover, the user receiver thermal noise is assumed to be negligible with the respect to $M_{u}^{i}(t)$ when the receiver experiences the urban environment effects. It could not be assumed negligible in case of open sky environment (high $C / N_{0}$, high elevation angle between receiver and satellite), when the multipath error is supposed to have low effects.

- the vehicle-reference station receiver clock difference, $\left(b_{u}(t)-b_{s t a}(t)\right)$, and the receivers' bias hardware term, $\left(h_{u}(t)-h_{s t a}(t)\right)$ are estimated together as a unique term called clock bias term, bh

- Multipath error and thermal noise are modelled as a single error term, $M N_{u}^{i}(t)$. 


\subsubsection{Multipath error component isolation from pseudorange residual difference method}

The last step of the proposed multipath error component isolation method consists in isolating each individual multipath error component in addition to the thermal noise components, $\widehat{M N}_{u}^{i}(t)$, [1], from the clock bias term, $b h_{u}(t)$. The isolation process is conducted by:

1- Estimating $b h_{u}(t)$ from the pseudorange residual difference terms, $\widehat{b h}_{u}(t)$

2- Removing/subtracting the estimated clock bias term, $\widehat{b h}_{u}(t)$, from each pseudorange residual difference, $\epsilon^{i}(t)$, to estimate each $\widehat{M N}_{u}^{i}(t)$, Figure 1 shows the complete scheme.

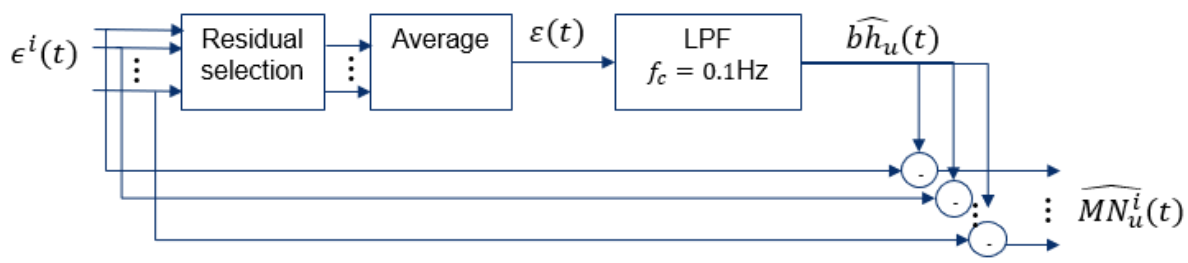

Figure 1 - Schematic of pseudo-range multipath error isolation from the residual containing multipath error, clock bias term and noise

In order to estimate $b h_{u}(t)$, a lowpass frequency filter could be applied to the any pseudorange residual difference term since this term has its power spectrum density around the lowest frequencies among all the pseudorange residual difference terms [1]. The filter proposed in this work is a first-order lowpass Butterworth filter with a cutoff frequency equal to $0.1 \mathrm{~Hz}[1]$.

Moreover, the clock bias term, $b h_{u}(t)$, is the same for all pseudorange residual differences belonging to the same time epoch whereas the multipath error and thermal noise components are different. Therefore, in order to reduce the impact of the receiver noise and multipath error components on the clock bias term estimate, $\widehat{b h}_{u}(t)$, the different satellite pseudorange residual differences per time epoch can be averaged together prior to the filtering process. However, this averaging process has to be performed with care when considering an urban scenario since some measurements might be strongly corrupted (for instance NLOS situations). Therefore, the averaging operation is only performed on a pseudorange residual difference subset where such a chosen subset is characterized by a high level of $C / N_{0}$. More specifically, only satellites with a $C / N_{0}$ higher than a certain threshold will be used for the clock bias term estimation process.

\section{MULTIPATH ERROR COMPONENT ISOLATION METHOD FOR DUAL CONSTELLATION}

In this section, the methodology proposed to isolate the pseudorange multipath error component from satellites of two different GNSS constellations in the L1 band, GPS and Galileo, is provided. In fact, the procedure to individually estimate the multipath error plus the thermal noise components for Galileo should theoretically be identical to the method proposed for GPS. However, if the estimation is to be jointly conducted, there are some additional issues to be considered.

The main interest in jointly using pseudorange measurements from different constellations is to improve the clock bias term estimation, $\widehat{b h}_{u}(t)$, due to the increase of the number of available pseudorange measurements being higher than the given $C / N_{0}$ threshold (see previous section). This improvement is quite interesting in urban environments where the number of NLOS satellites signals is higher and thus the number of available satellites over the $C / N_{0}$ threshold value per constellation is decreased. This is especially important for Galileo since the number of healthy constellation satellites is lower than for GPS. In fact, taking only into account Galileo, the satellites' subset over the given threshold can be empty in the worst case as was observed during the conducted data campaign. Therefore, it is impossible to obtain a precise and continuously over time clock bias term estimate for Galileo only measurements.

3.1 Pseudorange Multipath error component joint isolation method: GPS L1 C/A and Galileo E1 OS The main complication of the joint processing of GPS L1 C/A and Galileo E1 OS pseudorange measurements is the difference between the clock bias term estimate value between the two signals, $\delta(t)$ :

$$
\begin{gathered}
\epsilon_{G P S}^{i}(t) \approx b h_{u, G P S}(t)+M_{u, G P S}^{i}(t)+\eta_{u}^{i}(t)-\eta_{s t a}^{i}(t) \\
\epsilon_{G A L}^{i}(t) \approx\left(b h_{u, G P S}(t)+\delta(t)\right)+M P_{u, G A L}^{i}(t)+\eta_{u}^{i}(t)-\eta_{s t a}^{i}(t)
\end{gathered}
$$


$\delta(t)$ will be called from now on GPS to Galileo post-processing time-offset (GGPPTO). Different reasons explain this time-offset term, $\delta(t)(3-2)$,

$$
\delta(t)=\delta_{G G T O}(t)+\delta_{s t a}(t)+\delta_{u}(t)
$$

- Residual GPS-Galileo-Time-Offset, $\delta_{G G T O}(t)$ : There is an inherent time difference between the two constellation reference time systems which influence should be analyzed. First, both receivers should a priori provide the measurements with respect to GPS time and thus, the Galileo measurements should include this time offset. Second, the user receiver and reference station Galileo measurements are differenced and thus removes, as a consequence, all the constellation error terms. Therefore, it could be a priori concluded that this residual offset term should be removed. However, there is not enough information to guarantee that both receivers exactly time-tag the pseudorange measurements as explained before or following the same process. Therefore, it could be finally concluded that a small uncertainty remains.

- Reference station bias, $\delta_{s t a}(t)$ : the bias is composed by two different components,

- GPS-Galileo hardware bias: GPS L1 C/A is a BPSK(1) chip modulated signal whereas Galileo E1 OS is a $\operatorname{CBOC}(6,1,1 / 11)$ chip modulated signal. This means that the RF front-end filter introduces a different group delay for each filter that may and may not be corrected by the receiver before providing the pseudorange measurements. Additionally, there is no guarantee that the reference station hardware used for processing GPS L1 C/A signal is the same as the one used for processing Galileo E1 OS signal; which means that an additional bias reference station dependent could be introduced.

- GPS-Galileo processing bias: As stated before, the two signals have implemented a different chip modulation. Moreover, there is no information about the processing conducted by the user receiver or the reference station on these two signals. And this means that there is an additional time uncertainty between the processing of the two signals which could appear on the pseudorange measurments of each signal.

- User receiver bias, $\delta_{u}(t)$ : same as reference station bias,

- GPS-Galileo hardware bias.

- GPS-Galileo processing bias.

A possible solution applied in this work to jointly process GPS L1 C/A and Galileo E1 OS pseudorange measurements consists in:

1. Estimating the expected GGPPTO term, $\hat{\delta}$, for the duration of the pseudorange measurement data to be exploited (with another antenna in an open-sky environment).

2. To modify the Galileo E1 OS pseudorange measurements by subtracting the GGPPTO term

3. To jointly apply the multipath error component isolation method presented in 2.2 to the GPS L1 C/A and the modified Galileo E1 OS pseudorange measurements (clock bias term is estimated with GPS and Galileo measurements)

\subsection{GGPPTO estimation method}

The GGPPTO estimation method consists in:

1. Estimating the GPS L1 C/A clock bias term, $b h_{u, G P S}(t)$, using only GPS L1 C/A satellite measurements for a static receiver antenna in open-sky received signal conditions

2. Estimating the Galileo E1 OS clock bias term, $b h_{u, G A L}(t)=b h_{u, G P S}(t)+\delta(t)$, using only Galileo E1 OS satellite measurements for a static receiver antenna in open-sky received signal conditions

3. Differentiating the two to obtain a first raw estimation of the GGPPTO, $\hat{\delta}(t)$

4. Obtaining the final estimate of the GGPPTO as the mean of the raw GGPPTO, $\hat{\delta}$

$$
\hat{\delta}=E\left[b h_{u, G P S}(t)-b h_{u, G A L}(t)\right]
$$

Note that although it is probably not the case, the GGPPTO term is assumed to be a constant term and is estimated as such. Detailed reasons will be given in the next subsections, but the main reason is that the proposed method is not able to provide a reliable time-varying estimation.

Next sections are divided as follows: first, a detailed model of the GGPPTO term is presented. Second, the importance and order of magnitude of the GGPPTO is provided through different tests. Third, a detailed study of the different component affecting the GGPPTO is presented. Finally, a possible GPS to Galileo offset removal technique is presented. 


\subsubsection{GGPPTO model}

The GGPPTO model is equal to (derived from equations 3-1, 3-2 and Figure 1):

$$
\delta(t)=b \widehat{h_{u, G P S}}(t)-b \widehat{h_{u, G A L}}(t)
$$

The offset could be modelled as showed in 3-5

$$
\begin{gathered}
\delta(t)= \\
=F\left[\left(M_{u, G P S}(t)+\eta_{u, G P S}(t)+b h w_{u, G P S}(t)-M_{u, G A L}(t)-\eta_{u, G A L}(t)-b h w_{u, G A L}(t)+G G T O_{u}\right)-\right. \\
-\left(M_{s t a, G P S}(t)+\eta_{s t a, G P S}(t)+b h w_{s t a, G P S}(t)-M_{s t a, G A L}(t)-\eta_{s t a, G A L}(t)-b h w_{s t a, G A L}(t)\right. \\
\left.\left.+G G T O_{s t a}\right)\right]
\end{gathered}
$$

Where $F[\cdot]$ represents the lowpass filtering process presented in section 2.2 .3 . The resulting term is composed by:

a) The filtered difference of the receiver under test' residual multipath error component between GPS and Galileo measurements, $F\left[M_{u, G P S}(t)-M_{u, G A L}(t)\right]$.

b) The filtered difference of the receiver under test' residual thermal noise error between GPS and Galileo measurements, $F\left[\eta_{u, G P S}(t)-\eta_{u, G A L}(t)\right]$.

c) The filtered GPS L1 C/A to Galileo E1 OS receiver under test hardware/processing bias difference, $F\left[b h w_{u, G P S}(t)-b h w_{s t a, G P S}(t)\right]$.

d) The filtered difference of the reference station' residual multipath error component between GPS and Galileo measurements, $F\left[-\left(M_{s t a, G P S}(t)-M_{s t a, G A L}(t)\right)\right]$, which is no longer negligible with respect to the receiver under test multipath if the user receiver is in open sky environment.

e) The filtered difference of the reference station' residual thermal noise error between GPS and Galileo estimation, $F\left[-\left(\eta_{s t a, G P S}(t)-\eta_{s t a, G A L}(t)\right)\right]$.

f) The filtered GPS L1 C/A to Galileo E1 OS reference station hardware/processing bias difference, $F\left[-\left(b h w_{s t a, G P S}(t)-b h w_{s t a, G A L}(t)\right)\right]$.

g) Filtered difference between the filtered and observed receiver under test GPS-Galileo Time offset $G G T O_{u}$ and the filtered and observed reference station' GPS-Galileo Time offset GGTO sta, $F\left[G G T O_{u}-G G T O_{s t a}\right]$, also called residual GGTO, $F\left[\delta_{G G T O}\right]$. This term represents the potential different consideration of the GGTO between the receiver under test and reference station on the pseudorange measurements.

\subsubsection{GGPPTO relevance analysis}

To test the presence and the relevance of the GGPPTO, a static test in open-sky received signal conditions is conducted at ENAC to estimate the raw GGPPTO, $\hat{\delta}(t)$. A simultaneous data collection is performed with:

- High-quality receiver, NovAtel, with a dish antenna on the SIGNAV building rooftop

- A Mass-market receiver, U-Blox M8T b, sharing the same antenna as NovAtel

- A Mass-market receiver, U-Blox M8T a, using another antenna positioned on another building rooftop

And two different reference station are used to apply the multipath isolation method:

- $\quad$ The Reference station TLSE (position, x: 4627852.066 m, y: 119639.756 m, z: 4372993.324 m in RGF93 coordinates).

- $\quad$ The Reference station TLSG (position, x: 4628685.106 m, y: 119996.725 m, z: 4372110.023 m in RGF93 coordinates).

The $\hat{\delta}(t)$ estimation results obtained using the reference station TLSE are showed in Figure 2. As it can be seen, a significant offset is present, in each of the three different cases. Table 1 summarize the mean and the standard deviation of the resulting GPS to Galileo offsets. 


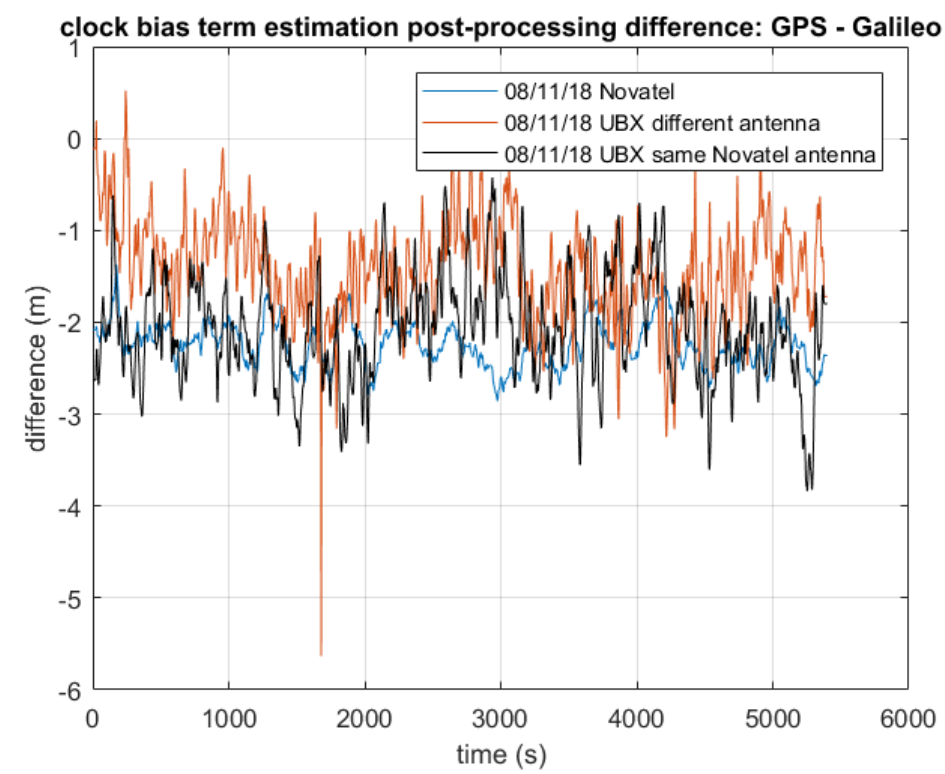

Figure 2 -GPS-to-Galileo Post-Processing Time Offset obtained from three different receivers, NoVatel, UBlox M8T $b$ which share the same antenna of Novatel and U-Blox M8T a which use a different antenna. The used reference station is TLSE

\begin{tabular}{|c|c|c|c|c|c|}
\hline & Mean (m) & Std (m) & & Mean $(\mathrm{m})$ & Std (m) \\
\hline TLSE & & & TLSG & & \\
\hline Novatel & -2.23 & 0.24 & Novatel & -0.29 & 0.25 \\
\hline UBX a & -1.41 & 0.54 & UBX a & 0.44 & 0.53 \\
\hline UBX b & -2.06 & 0.57 & UBX b & -0.13 & 0.56 \\
\hline
\end{tabular}

Table 1 - Mean and the standard deviation of the $\hat{\delta}(t)$ estimation obtained from three different receivers, NoVatel, U-Blox M8T b which shares the same antenna of Novatel and U-Blox M8T a which uses a different antenna. The used reference station are: 1) TLSE, 2) TLSG.

For TLSE results, the three different receiver presents a negative offset which is far from being negligible. The two different receivers which performs the measurements at the same time and share the same antenna have a similar offset mean. The standard deviation given by the NovAtel receiver is much smaller with respect the one given by the U-Blox receivers. For TLSG results, the three different receiver presents an offset which is close to 0 cannot be considered as equal to. For both reference stations, the Novatel and UBXb receivers have a similar offset mean.

In conclusion, the GGPPTO should be considered as a not negligible time offset which introduces a significant offset also in addition to smaller time-variations.

\subsubsection{GGPPTO estimation analysis}

In this section, the nature of the GGPPTO term is analyzed in order to determine which is the most suitable method to estimate its value: whether it is more adapted to just assume a constant value with an uncertainty estimation factor or whether the GGPPTO time-evolution can be estimated. In order to reach such a conclusion, several tests are conducted.

First, the influence of the reference station on the $\hat{\delta}(t)$ is analyzed. This analysis is conducted by performing a test which avoids the use of the receiver under test (Novatel or U-Blox): the same multipath isolation method proposed in the article is applied but using the TLSE reference station receiver as a receiver under test and TLSG as the reference station. Therefore, if the reference station has none to a small impact on the $\hat{\delta}(t)$, the estimated value should be almost zero. The $\hat{\delta}(t)$ for this test are presented below:

$$
\begin{gathered}
E\{\hat{\delta}(t)\}=-1.85 \mathrm{~m} \\
\sigma\{\hat{\delta}(t)\}=0.16 \mathrm{~m}
\end{gathered}
$$


The resulting mean is not zero and thus, it can be assumed that the reference stations introduces an offset which is an important contribution of the $\hat{\delta}(t)$ constant offset. This offset could be generated by the GPS-to-Galileo hardware/processing bias difference and/or the residual GGTO. Moreover, the obtained standard deviation, which should be a contribution of both reference stations sources of error (hardware/processing bias, residual GGTO, filtered multipath and noise), is lower than the standard deviation obtained for the three receivers under test (Novatel and U-Blox) cases (see Table 1).

Second, the contribution to the constant offset term of $\hat{\delta}(t)$ from the receiver under test is analyzed by conducting a new experiment: new GGPPTO values are estimated by two U-Blox receivers with different antennas positioned at the same location (SIGNAV rooftop building), under open sky environments with respect to the same reference station, TLSE. In this case, an additional $1^{\text {st }}$ order low-pass Butterworth filter with cutting frequency equal to 0.01 $\mathrm{Hz}$ is used to better observed the mean and the slow variations; besides this additional filter should remove any lingering multipath or noise contribution. The results are presented in Figure 3 and the estimated statistics in Table 2. From them, it can be observed that both estimates have the same very low rate evolving time offset; the difference of the two terms and the statistics are showed in Figure 4 and Table 3. Moreover, Figure 2 and Table 1 show the same behavior between different types of receivers, Novatel and UBXb. Therefore, it can be concluded that the more significant offset bias term is introduced by the reference station.

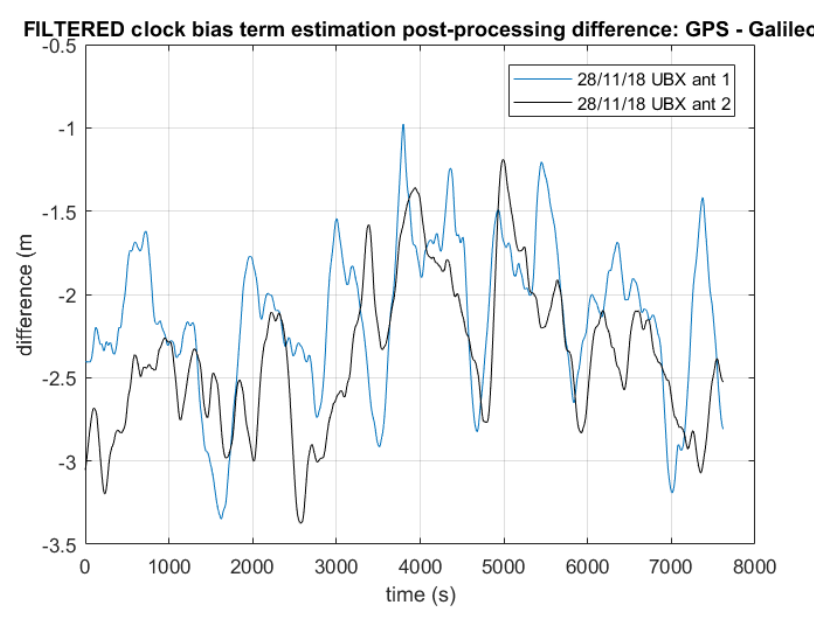

Figure 3 - The picture contains the GPS to Galileo offset obtained from two different U-Blox M8T receivers, which use different antennas. The used reference station is TLSE

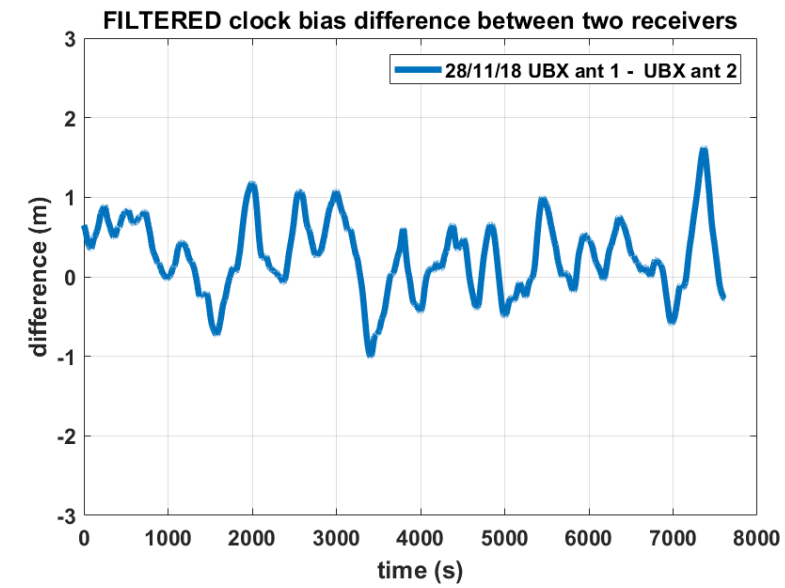

Figure 4 - The picture contains the difference between the GPS to Galileo offset obtained from two different U-Blox M8T receivers, which use different antennas. The used reference station is TLSE. Difference of terms plotted in Figure 3

\begin{tabular}{|c|c|c|}
\hline TLSE & Mean $(\mathrm{m})$ & Std $(\mathrm{m})$ \\
\hline UBX ant 1 & -2.12 & 0.4 \\
\hline UBX ant 2 & -2.37 & 0.38 \\
\hline
\end{tabular}

Table 2 - Table containing the mean and the standard deviation of GPS to Galileo offset of Figure 3

\begin{tabular}{|c|c|c|}
\hline TLSE & $\begin{array}{c}\text { Mean } \\
(\mathrm{m})\end{array}$ & $\begin{array}{c}\text { Std } \\
(\mathrm{m})\end{array}$ \\
\hline UBX ant 1 - UBX ant 2 & 0.25 & 0.4 \\
\hline
\end{tabular}

Table 3 - Table containing the mean and the standard deviation of Figure 3 
Third, the observed slow time-variations of the GGPPTO term, $\hat{\delta}(t)$, are analyzed. The contribution of the reference station is analyzed by estimating the $\hat{\delta}(t)$ with the same user receiver, Novatel, with respect to two reference stations, TLSE and TLSG, in open sky conditions. Moreover, an additional $1^{\text {st }}$ order low-pass Butterworth filter with cutting frequency equal to $0.01 \mathrm{~Hz}$ is applied to specifically inspect the contribution on the slow time variations. Figure 5 presents the $\hat{\delta}(t)$ time estimation and Table 5 presents the statistics. Moreover, the difference between the two terms and its statistics are showed, respectively, in Figure 6 and Table 4. From these two figures, it can be seen the high resemblance (plus and offset) between the two cases. Therefore, it can be concluded that the slow variations should mainly depend on the user receiver under test. This conclusion is verified from the analysis of Figure 4, where the standard deviation of the difference is equivalent to the individual standard deviations of each estimation; and this means that no uncertainty is removed from the knowledge of the estimation of $\hat{\delta}(t)$ from one receiver with respect to the other. To sum up, it can be concluded that the dominant term generating the GGPPTO slow time-variations is the receiver under test rather than the reference station.

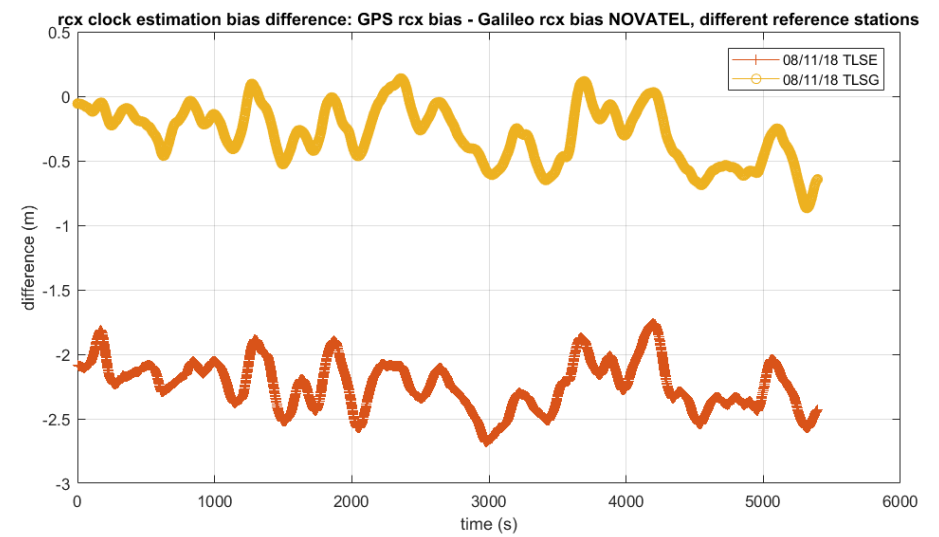

Figure 5 - Comparison of GGPPTO obtained used NovAtel and 1) TLSE, 2) TLSG

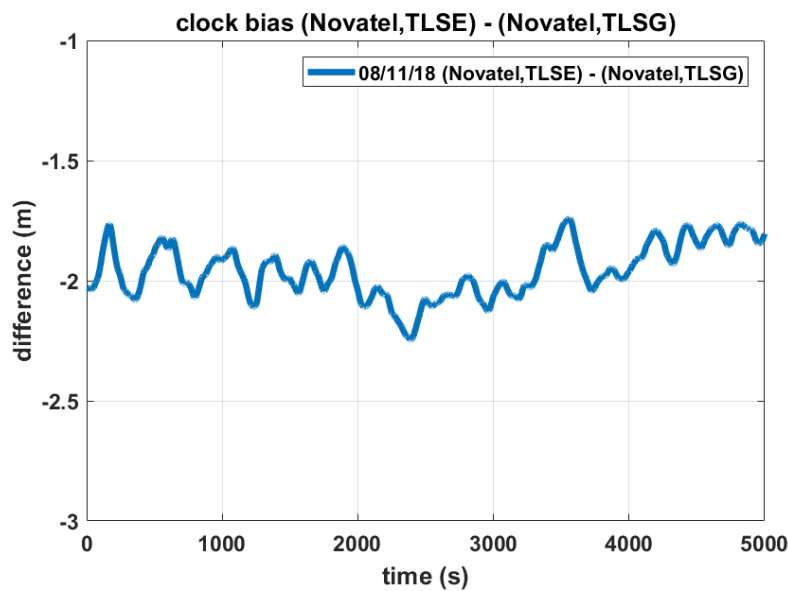

Figure 6 - The picture contains the difference between the GGPPTO obtained from (NovAtel - TLSE) and (NovAtel TLSG). Difference of terms plotted in Figure 3

\begin{tabular}{|c|c|c|}
\hline & $\begin{array}{c}\text { Mean } \\
(\mathrm{m})\end{array}$ & $\begin{array}{c}\text { Std } \\
(\mathrm{m})\end{array}$ \\
\hline $\begin{array}{c}\text { (NovAtel,TLSE) - } \\
\text { (NovAtel,TLSG) }\end{array}$ & -1.94 & 0.11 \\
\hline
\end{tabular}

Table 4 - Table containing the mean and the standard deviation of Figure 3

\begin{tabular}{|c|c|c|c|c|c|}
\hline & Mean (m) & Std (m) & & Mean (m) & Std (m) \\
\hline TLSE & & & TLSG & & \\
\hline Novatel & -2.23 & 0.19 & Novatel & -0.29 & 0.2 \\
\hline UBX a & -1.41 & 0.38 & UBX a & 0.44 & 0.37 \\
\hline UBX b & -2.06 & 0.38 & UBX b & -0.13 & 0.38 \\
\hline
\end{tabular}

Table 5 - Table containing the mean and the standard deviation of GPS to Galileo offset, comparisons between TLSE and TLSG reference stations

To summarize, it can be concluded that:

- Reference station introduces a non-negligible constant bias to the $\hat{\delta}(t)$ 
- The contribution of the receiver under test is more important than the reference station contribution to the $\hat{\delta}(t)$ time-variation

- Slow variation components of the $\hat{\delta}(t)$ appear to be created by the receiver under test

- Fast and slow time-variations created by the receiver under test are different between receivers

Therefore, it has been decided that the estimation of the GGPPTO term will provide a constant value in this work, $\hat{\delta}$, since the time evolution component of $\hat{\delta}(t)$ cannot be reliably predicted.

The proposed method to remove the GPS to Galileo offset in this work is:

- to use two different receivers during the data campaign. One emplied to get measurement in the urban scenario which will be characterized by the GPS to Galileo offset $\hat{\delta}^{[1]}(t)$, and the other in a static, open sky position in order estimate the GPS to Galileo offset $\hat{\delta}^{[2]}(t)$. If it is assumed that $\hat{\delta}^{[1]}(t)$ and $\hat{\delta}^{[2]}(t)$ have the same constant offset, and $E\left[\hat{\delta}^{[1]}(t)\right] \cong E\left[\hat{\delta}^{[2]}(t)\right]=\hat{\delta}$, the corrected residual difference for Galileo, $\overline{\epsilon_{G A L}^{l}}(t)$, is equal to

$$
\widetilde{\epsilon_{G A L}^{\tau}}(t)=\epsilon_{G A L}^{i}(t)-\hat{\delta}
$$

The complete proposed multipath isolation scheme, including the GGPPTO correction is presented in Figure 7:

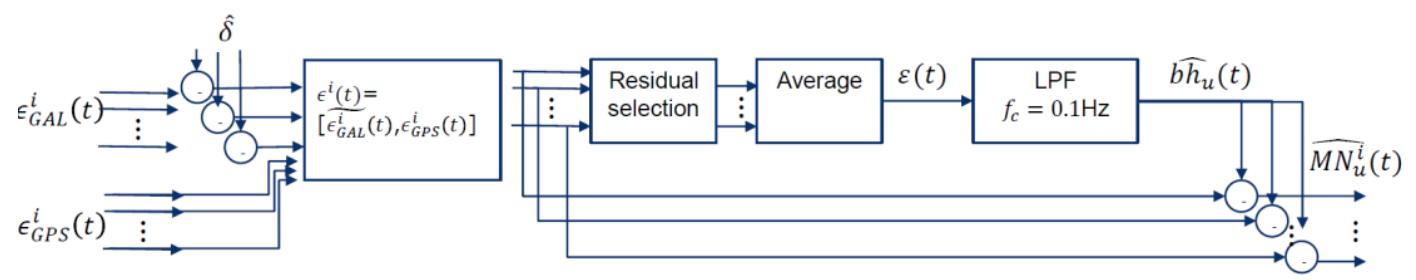

Figure 7 - Multi-constellation pseudo-range multipath error isolation method scheme.

\section{MULTIPATH ERROR COMPONENT CHARACTERIZATION}

Once the multipath error component, $\widehat{M N}_{u}^{i}$, is isolated, the multipath characterization process can be applied in order to obtain a mathematical model, which defines the multipath error component statistical behavior.

The multipath error component values are divided into different groups, where each group represents a specific received satellite signal reception condition, then, the characterization process is conducted per group. Three types of classifications are considered in this work, $C / N_{0}$, satellite elevation angle [1] and LOS/NLOS signal reception conditions. A justficatio of the choice of these three signal parameters is given below.

First, all the received satellites signals can be classified depending on the signal $C / N_{0}$ value. Moreover, the $C / N_{0}$ is an indicator used in the receiver position estimation which determines the quality of the received signal. Second, received satellite signals can be also classified depending on the signal elevation angle value. The elevation angle could be a good indicator of the multipath error component impact with respect to the LOS signal in the pseudorange measurement domain in the case of urban environment. Finally, the received satellite signals can be also classified depending on the LOS/NLOS signal reception conditions since the impact on the pseudorange measurement domain of the multipath error component should be quite different depending on whether the satellite LOS signal is received or not. Therefore, a different characterization for both situations should provide two different mathematical models/statistics which could be used to further improve the position calculation accuracy and reliability.

In this paper, an efficient approach for automatic LOS and NLOS satellite detection from sky images, taken from a fisheye camera pointing to the zenith mounted on the top of a car and synchronized with a GNSS receiver, is proposed. This approach is called sky-region satellite characterization.

The section is divided as follows. The first sub-section presents the motivations and the difficulties related to the image processing approach. The second sub-section presents the implemented core idea. The next sub-sections are devoted to, the image processing techniques and, at the end, the NLOS and LOS satellite classification method.

\subsection{Motivations and difficulties}

The main objective of the processing of the upward-facing camera images, which divides the acquired images into sky and non-sky regions, is first to allow the satellites classification into LOS and NLOS received signal conditions 
satellite classification and second, to use this separation to characterize the multipath error in two different models, one for NLOS and one for LOS satellites.

Dividing an image into sky and non-sky regions is not an easy task due to the variations of the environmental light, weather conditions, the facades of buildings (e.g. buildings with glass), etc. In fact, most of the conventional image segmentation algorithms initially developed for different purposes than outdoor navigation will produce poor results. Best results could be obtained in some specific circumstances (i.e. complete cloudy sky conditions) which could be exploited to perform some data measurements.

Finally, the proposed algorithm must also be effective with the type of images taken by the camera being used during the test campaign: grayscale with a JPEG compression; a possible output picture is shown in Figure 8.

\subsection{Main idea}

The most fundamental idea of the sky-region satellite characterization consists in:

- $\quad$ To implement an image processing technique determining which regions of the image are sky regions or non-sky regions.

- $\quad$ To implement a technique which projects the satellite inside the picture [6].

- To classify, depending on the satellite projection on the sky or non-sky regions of the processed image and thanks to having each picture synchronized to the vehicle GNSS receiver, the LOS and NLOS received satellite signal conditions.

\subsection{Image processing techniques}

This section presents the image processing techniques employed to identify the sky regions from the non-sky regions and the drawbacks of this technique. The proposed method is the object's edge enhancement approach followed by a flood filling algorithm.

\subsubsection{Object's edge enhancement and flood filling algorithm}

The object's edge enhancement is achieved in three different steps:

- $\quad$ Reduction of the presence of light variations modifying the luminance features of the picture.

- Application of a canny edge detector. The resulting picture is a black and white $(\mathrm{b} / \mathrm{w})$ picture where the edges are white. Figure 9 is the result of applying the detector to Figure 8.

- $\quad$ Flood filling process.

The flood filling process is explained next. First, the satellites are projected on the picture [6]. Second, the pixel corresponding to the location of the satellite with the highest carrier-to-noise ratio $\left(C / N_{0}\right)$ is considered to be a sky region. Third, from that projected point, the surrounding area is also considered as sky region until, in every possible direction, an edge is reached, as showed in Figure 10. Fourth, any area beyond these edges are assumed to be a non-sky region: the grey area in the Figure 10 is estimated as sky area while the remaining black and white areas are considered obstacles (non-sky) areas. Figure 11 shows a possible image processing sky-area estimation: the green points are considered in the sky-area, the red points are considered to be obstructed by obstacles.

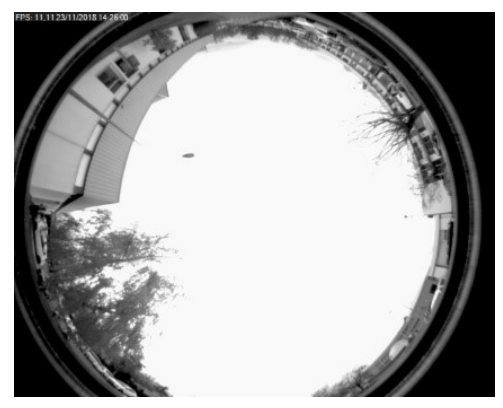

Figure 8 - uEye fish-eye camera output picture

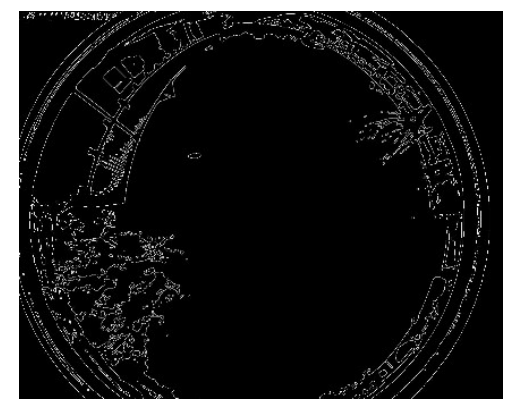

Figure 9 - Resulting picture from canny edge detector applied to Figure 8 


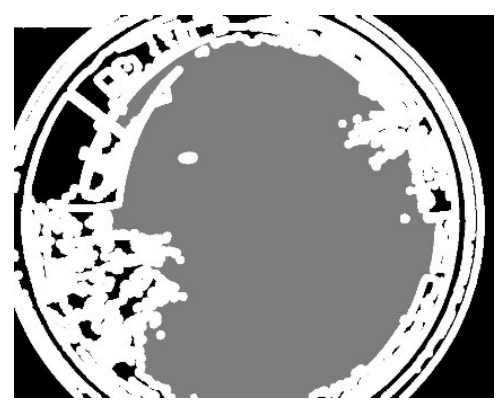

Figure 10 - Resulting picture from flood-filling algorithm

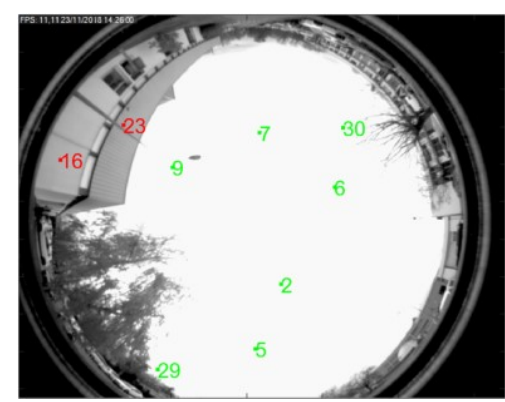

Figure 11 - Possible image processing sky-area estimation: the green points are considered in the sky-area, the red points are considered to be obstructed by obstacles

\subsubsection{Image Processing Drawbacks}

The main limitations of this identification method are the presence of image processing outcomings (erroneous borders detections, errors in the flood-filling operations, sun-glares impacting the pictures) and the presence of the systematic erroneous decisions (i.e. signal classified as NLOS if covered by trees, signal classified as LOS if the picture is shined by sun-glares). In the next paragraph the decision errors are explained in details.

The image processing decision based only on the simple sky-region detection described above could not characterize the real nature of the signal in the sequent cases:

a- Trees: trees act as an obstacle which may or may not cover the LOS between receiver and satellite (see Figure 12).

b- Sun-glares: sun-glares have a flash effect on the lens of the fish-eye camera. The image processing estimates the satellite in that portion of picture as LOS but it is impossible to estimate the nature of the signal from the image processing since this part of the image is lost (see Figure 13).

c- Shining buildings: Shining building refers to buildings highly reflecting sunlight. The image processing estimates the satellite in that portion of picture as LOS although it is obstructed by the building (see Figure 14).

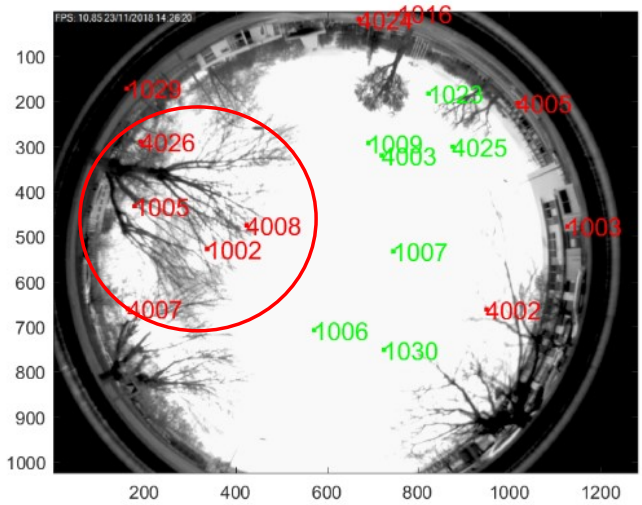

Figure 12 - Possible image processing NLOS/LOS estimation: the green points are considered in the sky-area, the red points are considered to be obstructed by obstacles 


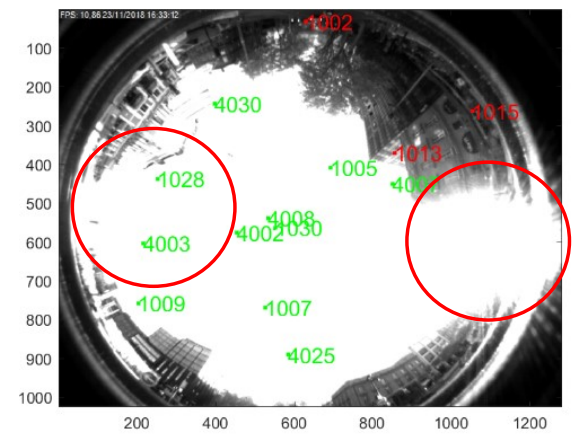

Figure 13 - Possible image processing NLOS/LOS estimation in presence of sun-glares.

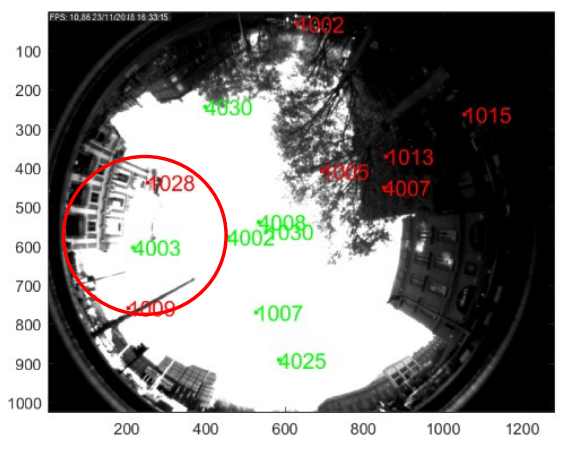

Figure 14 - Possible image processing NLOS/LOS estimation in presence of shining building.

Since these image estimation errors affect systematically the NLOS/LOS satellite classification by image processing algorithm only, the NLOS/LOS classification will have to be performed by using image processing but with any external aiding. The final algorithm should thus be able to remove the image processing errors to perform a reliable NLOS/LOS classification, using different parameters to check the image processing decision. Next section presents the final proposed NLOS/NLOS classification algorithm.

\subsection{LOS and NLOS decision algorithm:}

The final algorithm proposed to estimate whether the received signal is in LOS or NLOS conditions is based on the image processing techniques, on the satellite projection and on an external aiding introduced by the estimated $C / N_{0}$ of the received satellite signals. The algorithm is thus based on the following inputs:

1. Flood filled algorithm resulting picture.

2. Projection of the satellites $i$ position at given epoch $t$ with respect to the receiver antenna position, $\operatorname{pos}^{i}(t)$.

3. Carrier to noise ratio of the satellite $i$ at given time $t, C / N_{0}^{i}(t)$

The proposed algorithm is summarized in the Figure 15.
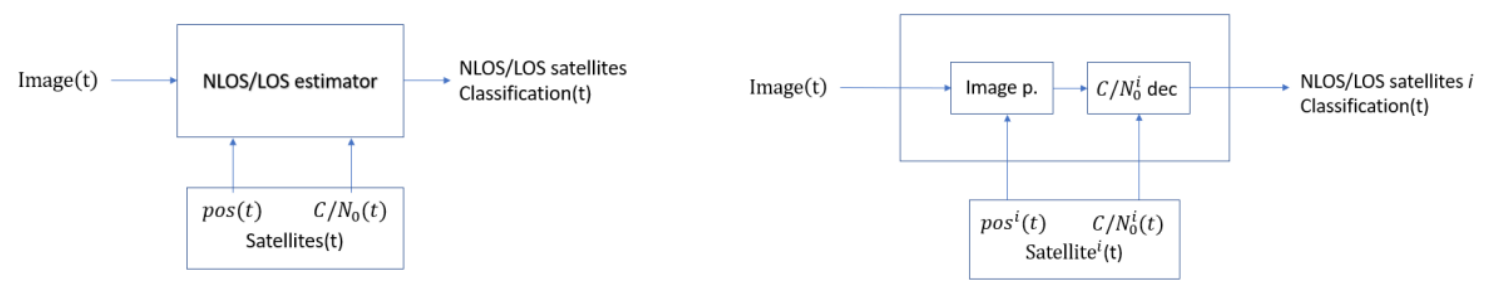

Figure 15 - Proposed LOS and NLOS decision algorithm

Based on these three inputs, the estimator procedure is the following. The estimator analyses the color of the pixel in which the satellite is projected [2] on the input pictures. Note that, if the pixel's color corresponds to sky area, the satellite is estimated as LOS satellite, otherwise it is estimated as NLOS satellite.

Then, the image processing estimation is checked by a $C / N_{0}$ threshold. From the results given in the result section 6 , in particular Table 6, it can be observed that in order to have a NLOS/LOS characterization less affected by image processing errors, a $C / N_{0}$ threshold equal to $35 \mathrm{~dB}-\mathrm{Hz}$ should be used with the following exclusion rules:

- In case of LOS image processing estimation:

- If $C / N_{0}^{i}(t)>35 \mathrm{~dB}-\mathrm{Hz}$, the estimation is confirmed as LOS estimation

- If $C / N_{0}^{i}(t)<35 \mathrm{~dB}-\mathrm{Hz}$, the estimation is considered wrong, probably due to image processing errors. It will not be used for the Multipath error NLOS/LOS characterization purpose

- In case of NLOS image processing estimation:

- If $C / N_{0}^{i}(t)>35 \mathrm{~dB}-\mathrm{Hz}$, the estimation is considered to be uncertain, probably due to trees error classification. It will not be used for the Multipath error NLOS/LOS characterization purpose

- If $C / N_{0}^{i}(t)<35 \mathrm{~dB}-\mathrm{Hz}$, the estimation is confirmed as NLOS estimation 
The equipment used during the data collection campaign consists of the following devices:
a. U-Blox M8T: GPS/Galileo receiver.
b. Novatel \& Span GPS/GLONASS receiver.
c. CNES Toulouse reference station receiver
d. IDS uEye Camera with fish-eye lens
e. Laptop used for the experiment
f. ENAC test vehicle used to do the experimental data campaign.

The role of the U-Blox M8T receiver in the experiment is to be the low-cost "mass-market" receiver working in the L1 frequency band which collects the data to be analyzed. The role of the NovAtel SPAN receiver is to obtain a very precise trajectory of the car during the data collection campaign which will be used as the true position of the receiver at any instant of time. Moreover, the SPAN receiver is also responsible for providing the vehicle heading information which can be extrapolated to the test receiver antenna using the known level-arm between IMU and the antenna. The SPAN receiver accuracy is at the decimeter-level or better [7].

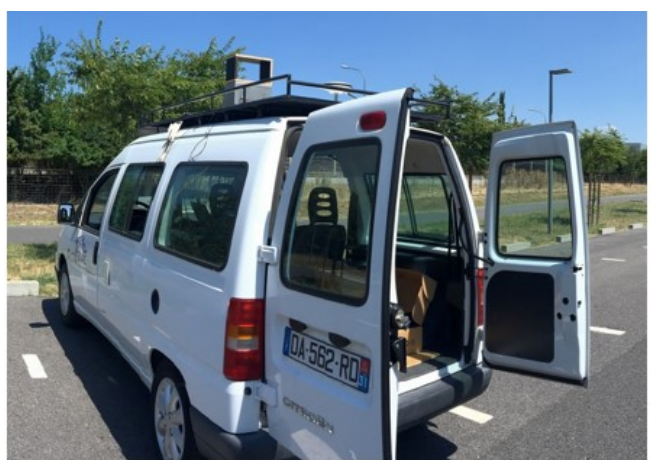

Figure 16- Picture of the vehicle used for the experiment

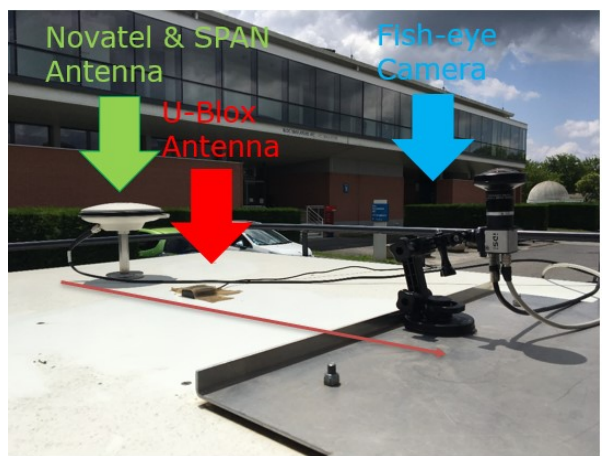

Figure 17- Rooftop of the vehicle, showing the UBlox, NovAtel antennas and the fish-eye camera

The reference station used in the experiment is the one located in the Toulouse site of the Centre National d'Etudes Spatiales (CNES). Figure 16 shows the test vehicle. Inside the van a small laboratory allows to work with the laptops and take the data campaign under control.

The role of the fish-eye camera consists in taking the pictures of the vehicle sky-environment in synchronization with the U-Blox estimated time. The fish-eye camera used in this study consists of a fish-eye lens attached to an IDS company CMOS sensor. The camera has a field angle greater than $180^{\circ}$ and captures images of the entire perimeter with an elevation angle range going from $0^{\circ}$ to $90^{\circ}$. The captured images are in a greyscale and have a resolution of $1280 \times 1024$ pixes. The internal parameters of the fish-eye camera were estimated with the Omnidirectional Calibration Toolbox for MATLAB [8].

\subsection{Set-up description}

In Figure 17, a picture of the equipment set-up mounted on the roof of the vehicle is presented. The U-Blox M8T antenna, NovAtel antenna and the uEye camera are installed on the roof of the ENAC test vehicle. The Novatel GNSS module, the IMU sensors, the consumer-grade GNSS receiver as well as the computers, which records the GNSS data, are inside the vehicle. A scheme of the physical set-up of these elements is shown in Figure 18 and Figure 19.

The data from U-Blox receiver and NovAtel SPAN receiver are synchronously collected. The Novatel \& Span collected information is synchronized with the U-Blox data by applying a GPS timestamp to the information. The SPAN receiver uses its internal memory to save the data measurements, as seen in Figure 18, which means that it is not directly connected to the laptop: the synchronization between the U-Blox, and NovAtel will thus be achieved in the post-processing stage.

The synchronization between the camera and the U-Blox receiver, Figure 20, is performed in the sequent way: the U-Blox is connected to the laptop which is able, through a software, to retrieve information on the U-Blox clock. Then, the clock of U-Blox receiver is used to drive the clock of the laptop. Therefore, a given image acquisition software installed on the laptop is used to acquire sequentially pictures with a time step of 1 second from the uEye camera. 


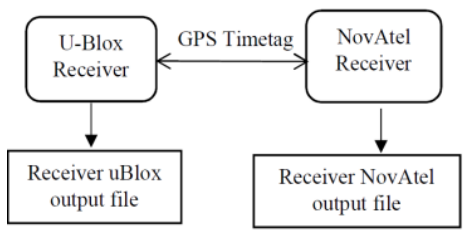

Figure 18 - U-Blox and NovAtel synchronization setup.

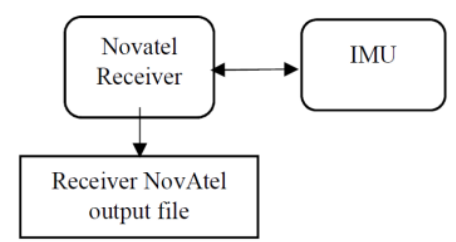

Figure 19 - NovAtel setup. GNSS receiver and IMU work simultaneously.

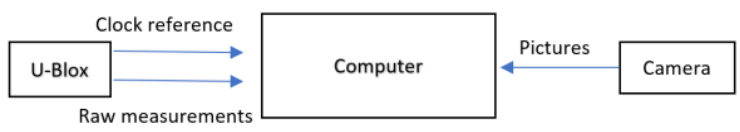

Figure 20 - U-Blox and uEye camera synchronization setup.

\subsection{Trajectory}

The trajectory followed in Toulouse urban area is divided in three different sections, ordered in the chronologically sequence.

The selected location for the data campaign was Toulouse urban area. The location was chosen in order to have a representation of different types of obstacles and different LOS/NLOS scenarios:

- the city center, with large and small streets around tall buildings and areas including bunch of trees;

- the suburbs; large and small streets in presence of small buildings;

- open areas close to the River.

\section{RESULTS}

The section presents the experimental results. The first section includes the data campaign parameters. In 6.1 the data campaign parameters are presented. The section 6.2 presents the expected behavior of the estimated multipath error depending on LOS and NLOS signal reception conditions. In 6.3 the preliminary multipath error model, characterized by $C / N_{0}$ and elevation angle is presented and commented. In section 6.4 the LOS multipath error model, characterized by $C / N_{0}$ and elevation angle, is presented and commented. Finally, in the section 6.5 the NLOS multipath error model, characterized by $C / N_{0}$ and elevation angle, is presented and commented.

\subsection{Data campaign parameters}

The following parameters were used to configure the data campaign equipment:

- $\quad$ The U-Blox receiver works in the L1 band, centered at $1575.42 \mathrm{MHz}$.

- The U-Blox's output data frequency is $1 \mathrm{~Hz}$.

- The evaluated constellation are GPS and Galileo.

- The Novatel SPAN output data frequency is $5 \mathrm{~Hz}$.

- The data collection's duration is roughly 3 hours and 10 minutes.

- Estimated GGPPTO, $\hat{\delta}=-2.25 \mathrm{~m}$.

\subsection{Expected behaviour of the estimated multipath error component depending on LOS and NLOS} signal reception conditions

The estimated multipath error component plus the remaining noise component pdf of a LOS satellite should theoretically be close to a centered Gaussian distribution. On the other hand, the absence of LOS signal should always introduce a positive bias in the pseudorange measurement: the receiver tracks the NLOS signal(s). Therefore, when taking into account the thermal noise component terms, the resulting multipath error component distribution should tend to have a very heavy positive tail. A more detailed explanation is given in [1].

\subsection{Preliminary Multipath error characterization with respect to $C / N_{0}$ and elevation angle classification} Before classifying the pseudorange measurements between NLOS and LOS signal reception conditions cases and before characterizing the two resulting multipath error and noise components subsets as a function of the received signal $C / N_{0}$ and the satellite elevation angle, the analysis of the characterization obtained from all the measurements without prior NLOS/LOS discrimination is presented. These results obtained from the whole dataset are presented next, focusing first in specific situations and presenting second the whole multipath error model. These results will also be used to determine the $C / N_{0}$ threshold used in the LOS and NLOS decision algorithm presented in section 4.4 . 
Figure 21 presents to the multipath error component pdf in the 45-50 dB-Hz $C \backslash \mathrm{N}_{0}$ range. The multipath error component has a symmetric Gaussian shape centered in 0 and a standard deviation of 0.82 meters. The pdf symmetry implies that the multipath error component under these signal reception conditions is mainly generated from LOS signals measurements as stated in section 6.2. Figure 22 presents to the multipath error component pdf in the 30-35 dB-Hz $\mathrm{C} \backslash \mathrm{N}_{0}$ range. The pdf seems to be a Non-Gaussian distribution. It can be seen that the total pdf is slightly non-symmetrical: the pdf's values corresponding to the positive multipath errors seem to be higher than the negative part. This phenomenon is probably due to the presence of signals received in NLOS conditions that result in positive biases as stated in section 6.2.

Figure 23 corresponds to the multipath error component pdf in the $50-60^{\circ}$ elevation angle range. The multipath error component has a symmetric Gaussian shape centered in 0.8 and a standard deviation of 4.68 meters. The pdf symmetry implies that the multipath error is mainly generated from LOS signals measurements. Figure 24 corresponds to the multipath error component pdf in the in the $10-20^{\circ}$ elevation angle range. In this picture, the probability density function is mostly positive biased; the pdf mean is equal to 10.24 meters. As well as before, the pdf positive error part is higher than the negative error part. However, a very important peak can be appreciated around 0 meters. Therefore, although the multipath error component pdf at this elevation angle seems to be generated by a significant number of NLOS satellites, it appears that a relevant quantity of LOS still is found.

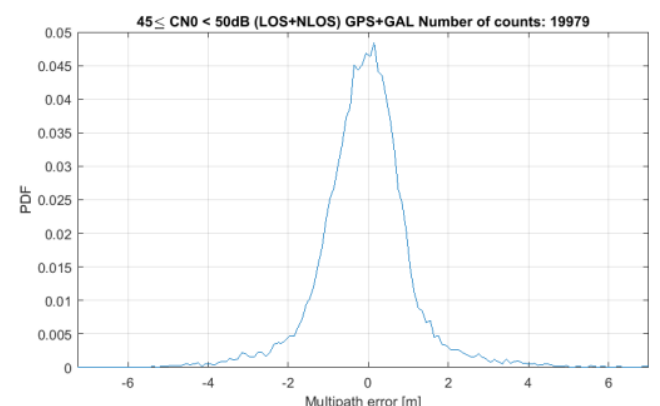

Figure 21 - Pdf of multipath error component in the 45-50 dB-Hz $\mathrm{C} \backslash \mathrm{N}_{0}$ band. GPS constellation case. $\mu=$ $0.06[\mathrm{~m}], \sigma=0.82[\mathrm{~m}]$

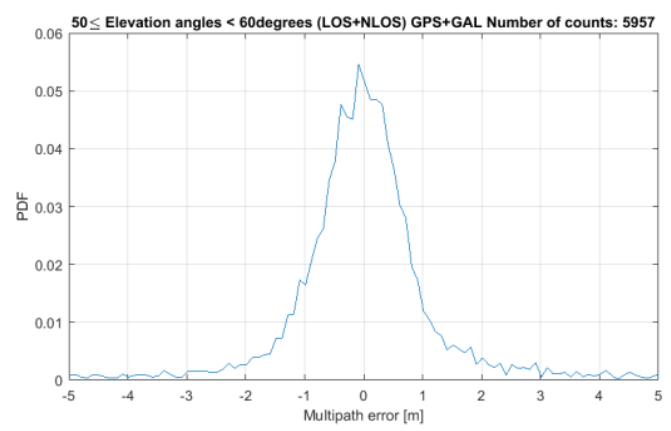

Figure 23 - Pdf of multipath error component in the 45-50 dB-Hz $C \backslash N_{0}$ band. GPS constellation case. $\mu=0.80[\mathrm{~m}], \sigma=4.68[\mathrm{~m}]$

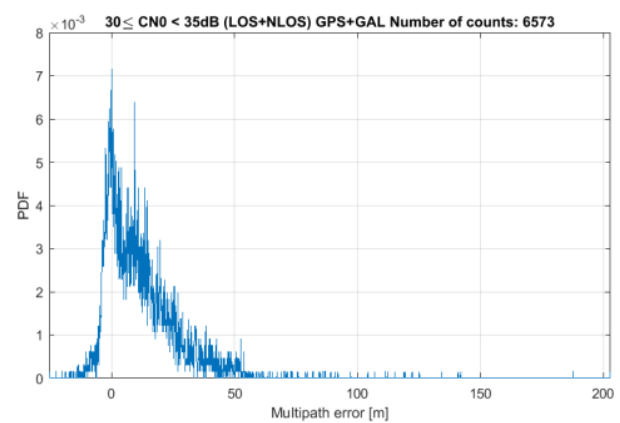

Figure 22 - Pdf of multipath error component in the 30-35 dB-Hz $C \backslash N_{0}$ band. GPS constellation case. $\mu=25.41[\mathrm{~m}], \sigma=21.29[\mathrm{~m}]$

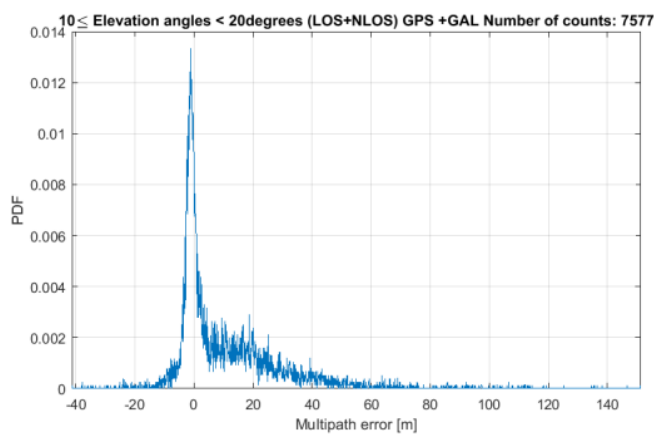

Figure 24 - Pdf of multipath error component in the 30-35 dB-Hz $C \backslash N_{0}$ band. GPS constellation case. $\mu=10.24[\mathrm{~m}], \sigma=18.09[\mathrm{~m}]$

Table 6 contains the pdf's mean and standard deviation values for each different pdf characterized by a different elevation angle range and $C / N_{0}$ range.

From this table it can be observed that:

1- From Table 6, it can be observed that from range 30-35 dB-Hz to lower values the mean is far from being equal to 0 . Therefore, it can be observed that from the $30-35 \mathrm{~dB}-\mathrm{Hz}$ range, a significant number of NLOS signals are received

2- The multipath error standard deviation decreases with the $C / N_{0}$ increase

3- $C / N_{0}$ parameter can be used to differentiate between LOS and NLOS signal conditions

a. Up to the 30-35 dB-Hz range, a significant number of NLOS signals are received

b. $C / N_{0}$ threshold of the NLOS/LOS decision algorithm is selected to be $35 \mathrm{~dB}-\mathrm{Hz}$.

4- The elevation angle does not appear to be a good NLOS/LOS discriminator:

a. Mean and standard deviation seems to be affected by relevant presence of NLOS also for high elevations. 
b. Even for low elevation angle values a significant number of LOS signals appear

\begin{tabular}{|c|c|c|c|c|c|c|c|c|c|c|c|c|}
\hline & \multicolumn{12}{|c|}{ Elevation angle range (degrees) } \\
\hline & \multicolumn{2}{|c|}{ 0-10 } & \multicolumn{2}{|c|}{$10-20$} & \multicolumn{2}{|c|}{$20-30$} & \multicolumn{2}{|c|}{$30-40$} & \multicolumn{2}{|c|}{$40-50$} & \multicolumn{2}{|c|}{$>\mathbf{5 0}$} \\
\hline $\begin{array}{c}C / N_{0} \\
\text { range } \\
\text { (dB-Hz) }\end{array}$ & $\begin{array}{c}\text { Mean } \\
(\mathrm{m})\end{array}$ & $\begin{array}{l}\text { Std } \\
(\mathrm{m})\end{array}$ & $\begin{array}{c}\text { Mean } \\
(\mathrm{m})\end{array}$ & $\begin{array}{l}\text { Std } \\
(\mathrm{m})\end{array}$ & $\begin{array}{c}\text { Mean } \\
(\mathrm{m})\end{array}$ & $\begin{array}{l}\text { Std } \\
(\mathrm{m})\end{array}$ & $\begin{array}{c}\text { Mean } \\
(\mathrm{m})\end{array}$ & $\begin{array}{l}\text { Std } \\
(\mathrm{m})\end{array}$ & $\begin{array}{c}\text { Mean } \\
(\mathrm{m})\end{array}$ & $\begin{array}{l}\text { Std } \\
(\mathrm{m})\end{array}$ & $\begin{array}{c}\text { Mean } \\
(\mathrm{m})\end{array}$ & $\begin{array}{l}\text { Std } \\
(\mathrm{m})\end{array}$ \\
\hline $0-5$ & - & - & - & - & - & - & - & - & - & - & - & - \\
\hline $5-10$ & $-9,41$ & 24,42 & 6,72 & 52,48 & & & 28,62 & 17,78 & 51,69 & 0,00 & - & - \\
\hline $10-15$ & $-22,94$ & 31,63 & 22 & 29,24 & 30,39 & 34,89 & 22,18 & 27,41 & 34,78 & 28,03 & 37,67 & 1,96 \\
\hline $15-20$ & 30,63 & 43,95 & 27,87 & 21,69 & 24,92 & 25,22 & 24,37 & 21,57 & 34,50 & 16,65 & 58,35 & 43,34 \\
\hline $20-25$ & 23,66 & 24,88 & 20,54 & 20,51 & 20 & 19,95 & 19,37 & 23,88 & 20,11 & 13,72 & 7,68 & 24,32 \\
\hline $25-30$ & 20,85 & 24,29 & 15,72 & 19,76 & 14,29 & 15,98 & 10,90 & 16,62 & 12,12 & 15,57 & 10,19 & 11,73 \\
\hline $30-35$ & 8,82 & 18,11 & 7,43 & 16,20 & 7,72 & 12,97 & 5,10 & 10,81 & 4,77 & 7,68 & 4,47 & 10,87 \\
\hline $35-40$ & 0,21 & 9,10 & 1,03 & 9,58 & 1,06 & 10,02 & 0,80 & 6,28 & 0,71 & 6,06 & $-0,66$ & 4,53 \\
\hline $40-45$ & $-1,62$ & 2,92 & 0,24 & 6,41 & $-0,44$ & 2,66 & $-0,38$ & 2,90 & $-0,36$ & 2,37 & $-0,43$ & 1,90 \\
\hline $45-50$ & $-1,87$ & 0,00 & $-0,73$ & 1,49 & $-0,26$ & 2,00 & $-0,55$ & 1,62 & $-0,19$ & 1,21 & 0,07 & 1,12 \\
\hline $50-55$ & - & - & - & - & $-0,84$ & 0,13 & 0,07 & 1,04 & $-0,09$ & 0,95 & 0,24 & 0,80 \\
\hline $55-60$ & - & - & - & - & - & - & - & - & - & - & 0,93 & 0,94 \\
\hline
\end{tabular}

Table 6 - Pseudorange multipath error component pdf's mean values and standard deviations characterized by a different elevation angle range and $C / N_{0}$ range

\subsection{LOS Multipath error characterization case}

This section presents the experimental results of the multipath error and noise components model characterized by $C / N_{0}$ and elevation angle for the subsets of satellites classified as received in LOS conditions during the data campaign using the discrimination algorithm presented in section 4.4.

Table 7 presents the mean and the standard deviation of the multipath error model for different $C / N_{0}$ bands and elevation angle bands. From this table, it can be clearly seen that the multipath plus noise components follow a Gaussian shape centered around 0 and that have a standard deviation which increases along the $C / N_{0}$ decrease. Moreover, it can be observed that the elevation angle does not impact the mean and, just for the lowest $C / N_{0}$ range, it may impact the standard deviation. The LOS Multipath Model confirms the expected centered Gaussian shape.

\begin{tabular}{|c|c|c|c|c|c|c|c|c|c|c|c|c|}
\hline & \multicolumn{10}{|c|}{ Elevation angle range (degrees) } \\
\hline & \multicolumn{2}{|c|}{$\mathbf{0 - 1 0}$} & \multicolumn{2}{|c|}{$\mathbf{1 0}-\mathbf{2 0}$} & \multicolumn{2}{|c|}{$\mathbf{2 0}-\mathbf{3 0}$} & \multicolumn{2}{|c|}{$\mathbf{3 0}-\mathbf{4 0}$} & \multicolumn{2}{|c|}{$\mathbf{4 0 - 5 0}$} & \multicolumn{2}{c|}{$>\mathbf{5 0}$} \\
\hline $\begin{array}{c}\boldsymbol{C} / \boldsymbol{N}_{\mathbf{0}} \\
\text { range } \\
(\mathbf{d B}-\mathbf{H z})\end{array}$ & $\begin{array}{c}\text { Mean } \\
(\mathrm{m})\end{array}$ & $\begin{array}{c}\text { Std } \\
(\mathrm{m})\end{array}$ & $\begin{array}{c}\text { Mean } \\
(\mathrm{m})\end{array}$ & $\begin{array}{c}\text { Std } \\
(\mathrm{m})\end{array}$ & $\begin{array}{c}\text { Mean } \\
(\mathrm{m})\end{array}$ & $\begin{array}{c}\text { Std } \\
(\mathrm{m})\end{array}$ & $\begin{array}{c}\text { Mean } \\
(\mathrm{m})\end{array}$ & $\begin{array}{c}\text { Std } \\
(\mathrm{m})\end{array}$ & $\begin{array}{c}\text { Mean } \\
(\mathrm{m})\end{array}$ & $\begin{array}{c}\text { Std } \\
(\mathrm{m})\end{array}$ & $\begin{array}{c}\text { Mean } \\
(\mathrm{m})\end{array}$ & $\begin{array}{c}\text { Std } \\
(\mathrm{m})\end{array}$ \\
\hline $\mathbf{3 5}-\mathbf{4 0}$ & $-0,1$ & 5,75 & 0.8 & 11,48 & 0,82 & 9,47 & 0,60 & 6,44 & $-0,15$ & 5,70 & $-0,88$ & 4,49 \\
\hline $\mathbf{4 0}-\mathbf{4 5}$ & $-0,22$ & 1,98 & 0,10 & 9,05 & $-0,52$ & 2,68 & $-0,35$ & 2,96 & $-0,38$ & 2,22 & $-0,42$ & 1,78 \\
\hline $\mathbf{4 5}-\mathbf{5 0}$ & $-0,87$ & 0,00 & $-0,77$ & 1,91 & $-0,13$ & 2,16 & $-0,51$ & 1,72 & $-0,17$ & 1,19 & 0,08 & 1,10 \\
\hline $\mathbf{5 0}-\mathbf{5 5}$ & - & - & - & - & - & - & 0,02 & 1,12 & $-0,06$ & 0,91 & 0,03 & 0,79 \\
\hline $\mathbf{5 5}-\mathbf{6 0}$ & - & - & - & - & - & - & - & - & - & - & 0,08 & 0,94 \\
\hline
\end{tabular}

Table 7 - Table containing the Line-of-Sight pseudorange multipath error component pdf's mean values and standard deviations characterized by a different elevation angle range and $C / N_{0}$ range

\subsection{NLOS Multipath error characterization case}

This section presents the experimental results of the multipath error and noise components model characterized by $C / N_{0}$ and elevation angle for the subsets of satellites classified as received in LOS conditions during the data campaign using the discrimination algorithm presented in section 4.4.

Table 8 presents the mean and the standard deviation of the multipath error model, for different $C / N_{0}$ bands and elevation angle bands. From this table, it can be seen, that the multipath error probability density function is mostly positive biased, and that these positive offsets increase with the $C / N_{0}$ decrease. At the same time, the standard deviation increases along the $C / N_{0}$ decrease. The positive pdf part of the MP error is therefore much higher than the negative one and increases with the $C / N_{0}$ decrease. Finally, it may be observed that the satellite elevation angle has an influence on the estimated mean and standard deviation for $C / N_{0}$ values above $10 \mathrm{~dB}-\mathrm{Hz}$. The NLOS Multipath Model confirms the expected Non-Gaussian positive biased shape 


\begin{tabular}{|c|c|c|c|c|c|c|c|c|c|c|c|c|}
\hline \multirow[b]{3}{*}{$\begin{array}{c}C / N_{0} \\
\text { range } \\
(\mathrm{dB}-\mathrm{Hz})\end{array}$} & \multicolumn{12}{|c|}{ Elevation angle range (degrees) } \\
\hline & \multicolumn{2}{|c|}{$\mathbf{0}-\mathbf{1 0}$} & \multicolumn{2}{|c|}{$10-20$} & \multicolumn{2}{|c|}{$20-30$} & \multicolumn{2}{|c|}{$30-40$} & \multicolumn{2}{|c|}{$40-50$} & \multicolumn{2}{|c|}{$>\mathbf{5 0}$} \\
\hline & $\begin{array}{c}\text { Mean } \\
(\mathrm{m})\end{array}$ & $\begin{array}{l}\text { Std } \\
(\mathrm{m})\end{array}$ & $\begin{array}{c}\text { Mean } \\
(\mathrm{m})\end{array}$ & $\begin{array}{l}\text { Std } \\
(\mathrm{m})\end{array}$ & $\begin{array}{c}\text { Mean } \\
(\mathrm{m})\end{array}$ & $\begin{array}{l}\text { Std } \\
(\mathrm{m})\end{array}$ & $\begin{array}{c}\text { Mean } \\
(\mathrm{m})\end{array}$ & $\begin{array}{l}\text { Std } \\
(\mathrm{m})\end{array}$ & $\begin{array}{c}\text { Mean } \\
(\mathrm{m})\end{array}$ & $\begin{array}{l}\text { Std } \\
(\mathrm{m})\end{array}$ & $\begin{array}{c}\text { Mean } \\
(\mathrm{m})\end{array}$ & $\begin{array}{l}\text { Std } \\
(\mathrm{m})\end{array}$ \\
\hline $5-10$ & 40,96 & 49,78 & 49,87 & 90,36 & - & - & 28,62 & 17,78 & 51,70 & 0,00 & - & - \\
\hline $10-15$ & 36,04 & 28,27 & 23,76 & 30,08 & 27,40 & 35,04 & 25,38 & 25,81 & 54,61 & 0,00 & - & - \\
\hline $15-20$ & 30,70 & 48,59 & 29,47 & 24,61 & 23,29 & 23,47 & 25,91 & 16,03 & 42,78 & 14,74 & - & - \\
\hline $20-25$ & 22,04 & 23,60 & 19,82 & 17,74 & 17,87 & 18,55 & 15,92 & 14,68 & 17,58 & 12,01 & 10,08 & 11,95 \\
\hline $25-30$ & 20,12 & 26,34 & 15,95 & 19,71 & 13,67 & 15,65 & 9,89 & 12,40 & 12,57 & 13,89 & 10,17 & 11,29 \\
\hline $30-35$ & 9,45 & 19,07 & 9,50 & 18,67 & 8,36 & 12,92 & 6,51 & 10,74 & 6,83 & 6,46 & 2,53 & 6,96 \\
\hline
\end{tabular}

Table 8 - Table containing the non-Line-of-Sight pseudorange multipath error component pdf's mean values and standard deviations characterized by a different elevation angle range and $C / N_{0}$ range

\section{CONCLUSIONS AND FUTURE WORK}

In this paper, a pseudorange multipath error component isolation methodology is proposed and applied to a dual constellation (GPS + Galileo) data collection obtained from a mass-market receiver in a moving vehicle in the Toulouse (France) urban area.

The proposed method does not differentiate between multipath error and thermal noise components; this means that the proposed method and the obtained results are in fact a joint estimate of both components in only one term.

A classification of the isolated multipath error component with the respect of the signal's reception conditions (NLOS/LOS) is proposed, using an upward-looking fish-eye camera and specific image-processing software to separate the satellite signals received in LOS and NLOS conditions; the classification is made by detecting which satellites are directly visible or hidden behind an obstacle. This technique has been further refined by the application of a $C / N_{0}$ threshold set to $35 \mathrm{~dB}-\mathrm{Hz}$ : signals with a lower $C / N_{0}$ than the thresholds are considered to be NLOS signals. This threshold has been set only for characterization purposes.

The LOS and NLOS isolated multipath error components have then been characterized by the calculation of their probability density function. The characterization is done with/without the application of the LOS/NLOS classification process. The isolated multipath error component values are classified depending on the received signal $\mathrm{C} / \mathrm{N}_{0}$ and the satellite elevation angle.

The main extracted conclusions from the different characterizations process are the followings. First the $C / N_{0}$ received signal parameter allows for a better classification of the multipath error component received signal conditions (LOS/NLOS) than the elevation angle. Second, for LOS signals having a $C / N_{0}$ over $40 \mathrm{~dB}-\mathrm{Hz}$, the standard deviation increases along the $C / N_{0}$ decreases irrespective of the satellite elevation angle while the mean remains always as a constant value around 0 meters. Third, for signals having a $C / N_{0}$ below $40 \mathrm{~dB}-\mathrm{Hz}$ and above $10 \mathrm{~dB}-\mathrm{Hz}$, the mean and the standard deviation decrease along the $C / N_{0}$ and the satellite elevation angle decrease.

The LOS/NLOS classification and the successive characterization have shown the different impact of the multipath error component on the pseudorange measurement as a function of some signal characteristics. Therefore, this finer characterization could be exploited in a PVT algorithm to increase the accuracy and reliability.

Future work will consist in:

- Characterize Multipath error model from Doppler measurements.

- Characterize Multipath error model from possible multipath correlation in distance.

- Taking advantage of the knowledge of the measurement pdf to optimize the navigation filter. 


\section{References}

[1] Eustachio Roberto Matera, Axel Javier Garcia Peña, Olivier Julien, Bertrand Ekambi. Characterization Of pseudorange Multipath Errors In An Urban Environment. ITSNT 2018, International Technical Symposium on Navigation and Timing, Oct 2018, Toulouse, France.

[2] D. J. R. Van Nee, "Multipath effects on GPS code phase measurements," NAVIGATION, Journal of Navigation, vol. 39, no. 2, 1992.

[3] J. M. Kelly, M. S. Braasch, and M. F. DiBenedetto, "Characterization of the effects of high multipath phase rates in GPS," GPS Solutions, vol. 7, no. 1, pp. 5-15, May 2003.

[4] B. M. Hannah, "Modelling and simulation of GPS multipath propagation" Ph.D. dissertation, Queensland University of Technology, Queensland, Australia, 2001.

[5] G. Moura and J. Castets, "Validation of deterministic simu- lation of GNSS reception in urban areas by comparison with measurement campaign," in Proc. of the European Navigation Conference GNSS, Toulouse, France, Apr. 2008.

[6] Bour, Houssam El Merabet, Youssef Ruichek, Yassine Messoussi, Rochdi Benmiloud, Ibtissam. An Efficient Sky Detection Algorithm From Fisheye Image Based on region classification and segment analysis. Transactions on Machine Learning and Artificial Intelligence

[7] A. El Idrissi, Y. El Merabet, Y. Ruichek, R. Touahni, A. Sbihi, C. Meurie, and A. Moussa. 2015. A MultipleObjects Recognition Method Based on Region Similarity Measures: Application to Roof Extraction from Orthophotoplans. (IJACSA) International Journal of Advanced Computer Science and Applications, Vol. 6, No. 11 (Novembre 2015).

[8] D. Scaramuzza et. al, “A Toolbox for Easy Calibrating Omnidirectional Cameras,” IEEE Int. Conf. on Intelligent Robots and Systems (IROS 2006), 2006. 